\title{
The Role of Fear-Avoidance Beliefs on Low Back Pain-Related Disability in a Developing Socioeconomic and Conservative Culture: $A$ Cross-Sectional Study of a Pakistani Population
}

This article was published in the following Dove Press journal:

Journal of Pain Research

\author{
Muhammad Naseeb Ullah \\ Khan (1D) \\ Natalie MV Morrison (iD) 2,3 \\ Paul W Marshall' \\ 'School of Health Sciences, Western \\ Sydney University, Sydney, NSW, \\ Australia; ${ }^{2}$ School of Medicine, Western \\ Sydney University, Sydney, NSW, \\ Australia; ${ }^{3}$ Translation Health Research \\ Institute, Western Sydney University, \\ Sydney, NSW, Australia
}

\begin{abstract}
Background: The relationship of low back pain, the world's top disabling condition, with functional disability is often explained by the mediation effect of fear, catastrophizing, and psychological distress. These relationships have not been explored within chronic back pain patients from a low socio-economic, predominantly Muslim country. Thus, it was unclear whether previously established pathways would be consistent in Pakistani pain patients to help guide Pakistani clinicians caring for back pain patients. This cross-sectional study translated English versions of questionnaires within the fear-avoidance model into Urdu, tested the clinimetric properties of the Urdu versions for people with chronic low back pain (CLBP) in Pakistan, and performed mediation analysis to investigate pathways of the fear-avoidance model.
\end{abstract}

Methods: Translation of questionnaires was completed in 4 steps using the forward-backward technique, with subsequent analyses for internal consistency (Cronbach's $\alpha$ ), construct validity (Pearson's $r$-value), and test-retest reliability (ICC $r$-value). Multiple mediation analysis with bootstrapping was performed to analyze pathways within the fear-avoidance model from the Urdu translated questionnaires.

Results: A total of 151 people from Pakistan with CLBP completed the questionnaires, with good results for internal consistency $(r>0.85)$, convergent validity $(r>0.59)$, and test-retest reliability (ICC $r>0.85)$. The association of pain with disability was significant $\left(B=2.36, r^{2}=\right.$ $0.19, p<0.001)$, and the indirect effect of the mediators explained $81 \%$ of pain intensity's total effect on disability. All mediators, apart from physical activity-related fear-avoidance beliefs, were significant mediators of the effect of pain intensity on disability.

Conclusion: The Urdu versions of the fear-avoidance questionnaires show good clinimetric properties for use in clinical settings and research in Pakistan. These analyses support existing data for the mediation effect of catastrophizing, psychological distress, and selfefficacy on pain-related disability, and extends these findings to suggest that fear about work may be more important in a relatively lower socioeconomic sample of pain patients.

Keywords: chronic low back pain, Urdu, mediation analysis, fear-avoidance, catastrophizing, Pakistan

\section{Introduction}

Chronic low back pain (CLBP) is a prevailing musculoskeletal issue that causes more functional impairment than any other condition. ${ }^{1}$ The financial consequences in western industrial countries are significant, with a collective approximate spending in direct and indirect medical care in the United States of America, Australia,
Correspondence: Muhammad Naseeb Ullah Khan

Tel +6I 246203915

Email dr.zyee@gmail.com
Journal of Pain Research 2020:13 2377-2387

2377 
and the United Kingdom reaching US\$100-150 billion yearly. $^{2}$ Critically, the problem of CLBP in countries experiencing economic and public healthcare development (eg, Pakistan, India, Sri Lanka, and Bangladesh) is not well understood. The prevalence of low back pain reported in South Asian countries (Pakistan 40\%, India 19\%, Sri Lanka $36 \%$, and Bangladesh 64\%) is much higher than the global prevalence rate. ${ }^{3}$ Indeed, the different societal structures found in these countries ${ }^{4}$ make it difficult to generalize the findings from more developed westernized counterparts, and, as a result of the low literacy rates and substandard occupational and health-care structures, the impact of CLBP might well be worse. ${ }^{5-7}$

Recent research has concentrated on the identification of factors termed "yellow flags" which initiate, exacerbate, and ameliorate pain and disability in CLBP. ${ }^{8}$ Psychological (cognitive, emotional, and behavioral) and social (cultural and socioeconomic) factors are considered significant determinants in the biopsychosocial approach of pain management. ${ }^{9}$ Studies of CLBP have demonstrated the significance of psychosocial determinants (eg, fear, catastrophizing, psychological distress, sense of helplessness and social or welfare support) in both explaining the relationship of pain with disability, ${ }^{10-11}$ and the changes that occur following different interventions (such as exercise and cognitive behavioral therapy). ${ }^{12,13}$ For example, the fear-avoidance model is commonly utilized within the biopsychosocial pain management approach to explain the relationship between exaggerated perceptions of pain and the avoidance of physical and social activities. ${ }^{14}$ According to Lethem et $\mathrm{al}^{14}$ the cognitive appraisal of pain develops not only from perceptions of discomfort received to the brain from a bodily region, but also from a secondary neural pathway, located exclusively within the brain, that is processing the emotional meaning of the discomfort. This neural pathway is idiosyncratic and is influenced by the past experiences of the individual. It is in this pathway that psychological discomfort, that is, the fear of pain itself (fear) or distress regarding the meaning of pain (catastrophizing), may be experienced, which leads an individual to actively avoid situations where there exists a potential for pain. Such avoidance further exacerbates the fear experience and ultimately increases disability.

A recent systematic review reported that the relationship between pain and disability was mediated by constructs within the fear-avoidance model including fear, catastrophizing, and psychological distress. ${ }^{11}$ One gap in this review was that the populations studied, and indeed subsequent research reporting quantitative evidence for the fear-avoidance model, are from predominantly AngloSaxon, liberal, and English-speaking countries. Indeed, findings supporting the fear-avoidance model are not consistent across all countries. For example, in contrast to the outcomes reported in the systematic review, several studies conducted amongst the Spanish population with CLBP consistently report no correlation between measures of fear or catastrophizing with pain and disability. ${ }^{15-17}$ Consequently, while pain education approaches appear to be effective for reducing pain-related disability in liberal and Anglo-Saxon cultures, ${ }^{18-20}$ a biomedical approach for CLBP has been more effective in Spain's LatinMediterranean culture. ${ }^{21,22}$ The conflicting evidence suggests that socio-cultural environment influences psychosocial factors; hence, findings confirming the fear-avoidance model in a western liberal society cannot be generalized to other cultures. However, while a number of studies have translated the well-established standard self-report questionnaires $^{23}$ for pain, disability, psychological risk factors (fear, catastrophizing), psychological distress (anxiety and depression), and self-efficacy into South American, ${ }^{24}$ African, ${ }^{25,26}$ European, ${ }^{27-29}$ and Asian languages ${ }^{30-32}$ idiomatically for cross-cultural adaptation and data comparisons, none of these studies quantified the pathways of the fear-avoidance model within the associated populations.

Pakistan, an economically developing low-income country, has a unique social and cultural setting. Funding for health care in Pakistan is limited, with expenditures estimated at $0.92 \%$ of GDP as reported by 2014 World Bank data. ${ }^{33}$ Occupational structures in Pakistan are mainly based on informal employment with very low accessibility to work insurance, compensation systems, and job modification. ${ }^{33}$ Indeed, thorough medical care coverage benefits at most $27 \%$ of the population which mostly consists of employees of government and armed forces; the other $73 \%$ are reliant on out-of-pocket payments. $^{34}$ Coping strategies for health issues in Pakistan are derived from Islamic doctrine which varies from the predominantly Judeo Christianity influenced liberal Anglo-Saxon culture found in the extant literature. Likewise, other factors including extended family support systems embedded in the eastern cultures and the role of the welfare system may influence the previously validated pathways in the fear-avoidance model. The role of family dynamics in health-related decision-making which 
subsequently influences biopsychosocial health outcomes in eastern cultures is crucial. ${ }^{4}$ No research has examined whether the relationship of pain with disability in people affected by CLBP in Pakistan is mediated by the proposed constructs of the fear-avoidance model, specifically fear, catastrophizing, and psychological distress.

We designed this research to provide empirical evidence of pathways in the fear-avoidance model in people with CLBP in Pakistan. The objectives of our study were to translate, culturally adapt, and analyze the psychometric properties of measures within the fear-avoidance model, and to investigate whether previously established relationships for the mediation effect of fear, catastrophizing, and psychological distress on pain-related disability were observed in people with CLBP in Pakistan.

\section{Materials and Methods Study Design and Participants}

This cross-cultural adaptation of questionnaires and crosssectional study was conducted in Pakistan with the data collected from CLBP sufferers between April 2019 and October 2019. Screening for inclusion in this study was ensured through an online survey tool. Out of 207 people screened, data from 151 participants were included in this study for analysis. Inclusion criteria were: non-specific CLBP (no pathoanatomical diagnosis) for more than 12 weeks in duration, with pain symptoms between coastal margins (T-12) and inferior gluteal folds, and, aged between 18 and 65 years. Participants were excluded from this study who had missing data/unanswered questions, reported recent surgery (ie, in the last 12 weeks), were pregnant during the past 12 months, or who had any current clinical diagnosis for a mental health condition, metabolic disorder, or disease (eg, diabetes). According to consensus-based standards for the selection of health measurement instruments (COSMIN) ${ }^{35}$ a sample size greater than 100 participants is commendable; as such, the final sample of 151 participants in this study was considered more than appropriate for further analysis (Table 1). Whereas, to achieve a statistical power of $0.8(p<0.05)$ for detection of anticipated mediation effect of a variable, the minimum sample size required is $71 .^{36}$

\section{Ethical Considerations}

This study was approved by the Human Research Ethics Committee of Western Sydney University (ethics approval \# H13097) and further ethical approval was sought from Multan medical college. Prior to proceeding with data collection, an information sheet detailing the research was provided to the participants and informed extended written consent was received from all the participants, for participation and publication of anonymous data, before commencing data collection.

\section{Translation Procedure}

Translation and cultural adaptation of CLBP-related selfreport questionnaires into the Urdu language was completed as per recommendations and guidelines. ${ }^{37}$ The forward-backward translation method was used for translation of measures and included the following steps: 1) English to Urdu forward translations; three professional native Urdu translators who were fluent in the English language, did the idiomatic translation of the measures to match the cultural perceptions; 2) translated versions of questionnaires were merged into single synthesized culturally adapted versions of questionnaires by a team consisting of an exercise professional, a translator, and a medical doctor; 3) Urdu to English back translation of synthesized versions was performed by a fourth professional translator; 4) the back translation was compared with original versions of questionnaires by an expert committee fluent in Urdu and English (two medical doctors, one exercise professional) for any inconsistencies and misinterpretations, of which none were found. Following the consolidation of Urdu versions of questionnaires, a pilot study was conducted with a small sample $(n=10)$ of Urdu speakers from Pakistan having back pain. The participants were also asked to comment on the understandability of the questions.

\section{Measures \\ Demographic Data}

Social and demographic characteristics of all participants were collected including age, gender, years of schooling, employment, and marital status via the online survey tool. All participants completed the following questionnaires.

\section{Pain}

The intensity of current self-rated low back pain was measured using the visual analog scale (VAS) on a 10point scale (extending from zero pain to worst pain). The VAS has been reported to have good construct validity and reliability. ${ }^{38}$

\section{Disability}

The Oswestry Disability Index (ODI) was utilized to assess self-reported functional disability due to CLBP. ${ }^{39}$ Version 2 of the ODI was used in this study which replaces 
Table I Demographic and Baseline Characteristics of Study Participants

\begin{tabular}{|c|c|c|c|}
\hline \multicolumn{2}{|l|}{$n=|5|$} & n (\%) & Mean (SD) \\
\hline \multicolumn{2}{|l|}{ Age (years) } & & $36.8(I 1.6)$ \\
\hline \multirow[t]{2}{*}{ Gender } & Female & $66(43.7)$ & \\
\hline & Male & $85(56.2)$ & \\
\hline \multicolumn{2}{|l|}{ Duration of Symptoms (years) } & & $4.9(3.3)$ \\
\hline \multirow[t]{4}{*}{ Employment status } & Full-time & $44(29.1)$ & \\
\hline & Part-time & $14(9.2)$ & \\
\hline & Unemployed & $48(31.7)$ & \\
\hline & Homemaker & $45(29.8)$ & \\
\hline \multirow[t]{4}{*}{ Education (years of schooling) } & $\leq 10$ years & $63(41.7)$ & \\
\hline & 12 years & $19(12.5)$ & \\
\hline & 14 years & $22(14.5)$ & \\
\hline & $\geq 16$ years & $47(31.1)$ & \\
\hline \multirow[t]{2}{*}{ Marital status } & Married & $125(82.7)$ & \\
\hline & Not married & $26(17.2)$ & \\
\hline \multicolumn{2}{|l|}{ Oswestry disability index (ODI, 0-100\%) } & & $34.6(17.4)$ \\
\hline \multicolumn{2}{|c|}{ Pain intensity (VAS, score range: $0-10 \mathrm{~cm}$ ) } & & $6.4(2.4)$ \\
\hline \multicolumn{2}{|c|}{ Fear avoidance beliefs - physical activity (FABQ-physical activity, score range: 0-24) } & & $17.2(5.8)$ \\
\hline \multicolumn{2}{|c|}{ Fear avoidance beliefs - work (FABQ-work, score range: 0-42) } & & $26.3(12.1)$ \\
\hline \multicolumn{2}{|c|}{ Pain catastrophizing (PCS, score range: $0-52$ ) } & & $33.9(13.7)$ \\
\hline \multicolumn{2}{|c|}{ Anxiety (HADS-anxiety, score range: $0-2 \mathrm{I}$ ) } & & $8.5(3.4)$ \\
\hline \multicolumn{2}{|c|}{ Depression (HADS-depression, score range: $0-2 I$ ) } & & $8.0(3.4)$ \\
\hline \multicolumn{2}{|c|}{ Functional self-efficacy (FSE, score range: 0-72) } & & $52.0(15.3)$ \\
\hline \multirow[t]{2}{*}{ Health-related Quality of Life (SF-12) } & Physical (PCS-12, score range: $0-100$ ) & & $39.6(6.6)$ \\
\hline & Mental (MCS-12, score range: $0-100)$ & & $39.8(7.6)$ \\
\hline
\end{tabular}

Abbreviations: FABQ, Fear Avoidance Beliefs Questionnaire; FSE, Functional Self-Efficacy Scale; HADS, Hospital Anxiety and Depression Scale; ODI, Oswestry Disability Index; PCS, Pain Catastrophizing Scale; SF-12 (PCS), Short form-12 questionnaire (physical component scale); SF-12 (MCS), Short form- 12 questionnaire (mental component scale); SD, standard deviation; VAS, visual analog scale.

the "sex life" subscale with "social life" due to low response rates to the former during pilot testing. ${ }^{39}$ Disability is expressed as a percentage, with higher scores indicating more severe disability.

\section{Fear-Avoidance Beliefs}

The evaluation of beliefs regarding the potentially damaging effects of physical activity and work on a person's CLBP was completed with the Fear Avoidance Beliefs Questionnaire (FABQ). ${ }^{40}$ Two subscales have been identified in FABQ to examine avoidance beliefs about work (FABQ-w; 0-42) and beliefs regarding physical activity (FABQ-pa; 0-24). The internal consistency of the FABQ subscales is $\alpha=0.77$ and 0.88 , respectively, and reliability is $r=0.95 .^{41}$ FABQ has been translated and validated into more than a dozen languages with good outcomes of internal consistency, convergent validity, and test-retest reliability. ${ }^{24-32}$

\section{Catastrophizing}

The pain catastrophizing scale (PCS) was created to examine the catastrophic feelings and notions of helplessness of pain patients. ${ }^{42}$ The overall reliability and internal consistency of the PCS is high. ${ }^{43,44}$ The PCS is divided into 3 subscales of helplessness ( 4 items), rumination (6 items), and magnification ( 3 items). The 13-items of the PCS are scored on a scale of 0 to $5(0=$ not at all, $4=$ all the time) where a high total score (out of a possible 52) signifies greater catastrophic cognitions.

\section{Anxiety and Depression}

The Hospital Anxiety and Depression Scale (HADS) contains 14 items divided into two subscales of anxiety (HADS-a, 7 items) and depression (HADS-d, 7 items). ${ }^{45}$ The statements are ranked on a scale from 0 to 3 , and higher anxiety or depression is indicated by a higher score. Several past studies have reported the translation and validation of HADS into various languages with high clinimetric outcomes. ${ }^{46}$ 


\section{Self-Efficacy}

We used the functional subscale of the Chronic Pain SelfEfficacy Scale ${ }^{47}$ to rates the confidence of participants regarding various activities. The modified version was used ${ }^{44}$ which assesses confidence for 9 items (such as walking $800 \mathrm{~m}$, lifting a $4 \mathrm{~kg}$ box, work/homemaker duties) on a scale of 0 (totally unconfident) to 8 (totally confident) while taking into account their current back pain. The maximum score was 72 , with higher scores pointing out greater confidence in the ability to perform different functions with current pain levels. The scale exhibits good internal consistency $(\alpha=0.88)$, and test-retest reliability (ICC $r=0.88,95 \% \mathrm{CI}=0.80-0.93){ }^{48,49}$

\section{Health-Related Generic Quality of Life (HRQoL)}

Short form 12 health survey (SF-12) ${ }^{50}$ was used to assess HRQoL. SF-12 has two subscales, physical component summary (PCS-12) and mental component summary (MCS-12). The scales are scored on a range of 1 to 100 and a higher score indicates better perceived health quality. In a population having back pain, SF-12 has shown good psychometric characteristics (PCS-12: $\mathrm{a}=0.77, \mathrm{MCS}-12$ : $\mathrm{a}=0.80){ }^{51}$

\section{Statistical Analysis}

All analyses were performed using SPSS (v25, IBM, USA). Internal consistency was analyzed using Cronbach's $\alpha$ coefficients for each questionnaire. Values between 0.70 and 0.95 are considered statistically significant while values lower than 0.30 indicated the response to an item to be unusual with the item potentially evaluating an alternative construct. ${ }^{52}$

Convergent construct validity was assessed by comparison of the correlation between related measures using Pearson's correlation coefficient because of an unavailability of gold standard measures for LBP in the Urdu language. A sub-sample of participants $(n=25)$ were asked to complete the online survey for a second time 12 weeks following their initial completion for test-retest reliability analysis using intraclass correlation coefficients.

Multiple mediation analyses were conducted to investigate the mediating influence of fear-avoidance beliefs, catastrophizing, anxiety, depression, HRQoL, and self-efficacy on the association of pain with disability in the Pakistani study population. ${ }^{53,54}$ Factors that were not correlated with either pain (VAS) or disability (ODI) scores according to the bivariate correlation matrix, or with $r$-value $>0.85$ indicating multicollinearity, were not included in mediation, as recommended for multivariate analysis. $^{55}$

Direct and indirect pathways of the mediation model 4 (Figure 1) were analyzed using a custom macro (PROCESS v3.4; processmacro.org) with 95\% confidence interval and bias-corrected bootstrapping (5000 resamples) to estimate the indirect effect. ${ }^{50}$ The following steps were needed to be fulfilled to confirm mediation: (1) VAS was

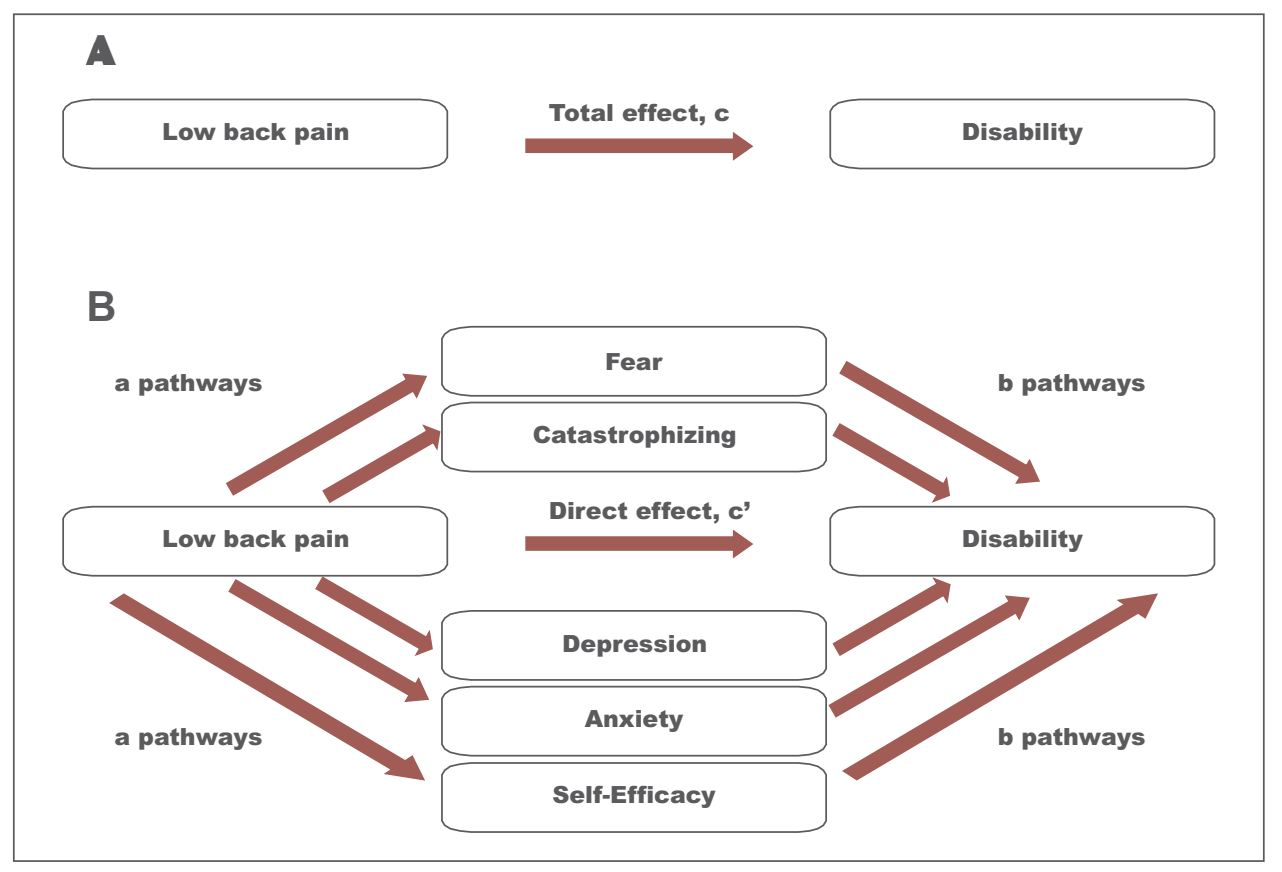

Figure I Example of mediation model analyzed in this study. (A) is the association of pain and disability (total effect c) and (B) are pathways of indirect effect of mediators. 
significantly correlated with ODI (total effect pathway, c path); (2) VAS was significantly correlated with all the proposed mediating variables (a pathways); (3) controlling for VAS, each of the mediating variables was significantly correlated with ODI (b pathways); and (4) the association between VAS and ODI (direct effect, c pathway) was reduced when mediating variables were taken into account (indirect effect, a $\mathrm{x}$ b pathways), with the $95 \%$ confidence interval (CI) for the indirect effect of each mediating variable outside of 0 . The significance level of this study was $p \leq 0.05$.

\section{Results}

Table 1 lists the baseline characteristics of the study participants. A total of 207 participants were screened for this study, and data collected from 151 participants were complete and eligible for further analysis. Participants with incomplete responses and who met the exclusion criteria of this study were omitted from the analysis. Out of 151 participants included in this study, 85 (56.2\%) were males and $66(43.7 \%)$ were females. The median age was 36.84 $(+11.5)$ years and the average duration of low back pain symptoms was $4.91(+3.31)$ years. One hundred and ten (72.8\%) participants were taking medication for pain relief. Work status was recorded in four categories: a) full-time $44(29.1 \%)$, b) part-time $14(9.2 \%)$, c) unemployed $48(31.7 \%)$ and d) homemaker $45(29.8 \%)$.

\section{Reliability and Validity of Measures}

The translated questionnaires were easy to comprehend. The data included in this study had no unanswered questions and no ambiguities were found in the understanding of questions. The internal consistency of the questionnaires was excellent, indicated by high values of Cronbach's coefficients (Table 2). No items from the questionnaires were inconsistent or considered for removal except FABQ-p (item 1) and FABQ-w (items 8, 13, 14, and 16). Multicollinearity was not found among the items of the scales.

The test-retest reliability of all scales was high, with average measures Intraclass Correlation Coefficient $r$-values ranging between 0.85 and 0.98 (Table 3). Correlation examining convergent validity was moderate to high (Table 4). Stronger correlations were found between measures of fear and catastrophizing with pain (FABQ-pa $r=0.627 p<0.001$, FABQ-w $r=0.690 p<0.001$, PCS $r=0.684 p<0.001$ ) than with disability (FABQ-pa $r=0.240 \quad p<0.001, \quad$ FABQ-w $r=0.474 \quad p<0.001$, PCS $r=0.411 p<0.001)$. Whereas, psychological distress had stronger correlations with disability (HADS-a $r=0.468$ $p<0.001$, HADS-d $r=0.441 p<0.001)$ than with pain (HADS-a $r=0.263 p<0.001$, HADS-d $r=0.231 p<0.001$ ). Correlations between FABQ-pa, FABQ-w, and PCS were high (ranging $r=0.590$ to $0.727 p<0.001$ ). Floor and ceiling effect were not observed.

\section{Effects of Psychosocial Factors}

Disability (measured with the ODI) was correlated with all psychosocial variables, whereas pain intensity (measured with the VAS) was correlated with each psychosocial factor except the physical component of SF-12 (PCS-12). Therefore, the SF-12 physical component (PCS-12) was excluded from further analysis. The Pearson's correlation coefficients (Table 4) were below 0.85 , thus multicollinearity was not detected. The regression model explained $45 \%(p<0.001)$ of the variance in ODI (disability) by VAS (pain) and the mediating variables. The association between pain intensity and disability was significant (c pathway, total effect: $B=2.36, r^{2}=0.19, p<0.001$ ) (Table 5). The indirect effect of the psychosocial mediators was $B=1.82(95 \% \mathrm{CI}=0.51$ to 3.32$)$, which explains $77.2 \%$ of the total effect. Indirect effects for the proposed mediators are depicted in Table 6. All of the proposed mediators apart from FAB-pa and MCS-12 fulfilled the criteria for significant mediation of the association of pain with disability.

\section{Discussion}

This is the first study to examine the psychometric characteristics of Urdu translated versions of measures of the fear-avoidance model, and to examine the pathways within the model in Pakistan's unique sociocultural environment. The main findings of this cross-sectional analysis of Pakistani CLBP sample were 1) Urdu translated versions of the Fear Avoidance Beliefs Questionnaire (FABQ), Pain Catastrophizing Scale (PCS), Oswestry Disability Scale (ODI), Hospital Anxiety and Depression Scale (HADS), Functional Self-Efficacy Scale (FSE), and Short form 12 (SF-12) had good internal consistency ( $\alpha=0.70-0.95$ ), test-retest reliability $(r<0.85)$ and convergent construct validity with no ceiling and floor effects; 2) fear-avoidance beliefs about work, catastrophizing, anxiety, depression, and self-efficacy explained the relationship between pain and disability while fear-avoidance beliefs about physical activity did not. 
Table 2 Reliability Statistics for Urdu Versions of Questionnaires

\begin{tabular}{|l|l|l|l|l|}
\hline Measure & $\begin{array}{l}\text { Cronbach's } \\
\text { Alpha }\end{array}$ & $\begin{array}{l}\text { Cronbach's Alpha Based on Standardized } \\
\text { Items }\end{array}$ & $\begin{array}{l}\text { No. of Items } \\
\text { in Measure }\end{array}$ & $\begin{array}{l}\text { Mean Inter-Item } \\
\text { Correlations }\end{array}$ \\
\hline ODI & 0.848 & 0.848 & 10 & 0.36 \\
FABQ & 0.958 & 0.957 & 16 & 0.58 \\
FABQ-physical activity & 0.877 & 0.877 & 4 & 0.64 \\
FABQ-work & 0.943 & 0.944 & 7 & 0.71 \\
PCS & 0.965 & 0.965 & 13 & 0.68 \\
PCS-Helplessness & 0.938 & 0.937 & 6 & 0.71 \\
PCS-Magnification & 0.887 & 0.889 & 3 & 0.73 \\
PCS-Rumination & 0.897 & 0.897 & 4 & 0.68 \\
HADS-anxiety & 0.688 & 0.687 & 7 & 0.24 \\
HADS-depression & 0.686 & 0.687 & 7 & 0.24 \\
FSE & 0.941 & 0.943 & 9 & 0.65 \\
\hline
\end{tabular}

Abbreviations: FABQ, Fear Avoidance beliefs questionnaire; FSE, Functional Self-Efficacy Scale; HADS, Hospital Anxiety and Depression Scale; ODI, Oswestry Disability Index; PCS, Pain Catastrophizing Scale.

Table 3 Test-Retest Reliability Statistics

\begin{tabular}{|c|c|c|c|c|c|}
\hline Measure & ICC & $95 \% \mathrm{Cl}$ & $p$-value & Test-I Mean (SD) & Test-2 Mean (SD) \\
\hline ODI & 0.98 & 0.95 to 0.99 & $<0.001$ & $27.6(15.8)$ & $27(17.4)$ \\
\hline FABQ-physical activity & 0.90 & 0.78 to 0.96 & $<0.001$ & $19.1(4.5)$ & $19.5(5.3)$ \\
\hline FABQ-work & 0.85 & 0.67 to 0.94 & $<0.001$ & $23.9(14.1)$ & $21.7(11.1)$ \\
\hline PCS & 0.95 & 0.88 to 0.98 & $<0.001$ & $26.9(9.5)$ & $26.6(12.1)$ \\
\hline HADS-anxiety & 0.89 & 0.69 to 0.95 & $<0.001$ & $8.1(2.8)$ & $7(3.6)$ \\
\hline HADS-depression & 0.89 & 0.75 to 0.95 & $<0.001$ & $6.4(3.3)$ & $6.5(3.4)$ \\
\hline FSE & 0.91 & 0.80 to 0.96 & $<0.001$ & $51.2(15.8)$ & $51(17.1)$ \\
\hline
\end{tabular}

Abbreviations: $\mathrm{Cl}$, confidence interval; FABQ, Fear Avoidance Beliefs Questionnaire; FSE, Functional Self-Efficacy Scale; HADS, Hospital Anxiety and Depression Scale; ICC, intraclass correlation coefficient; ODI, Oswestry Disability Index; PCS, Pain Catastrophizing Scale; SD, standard deviation.

Table 4 Correlations (r-Value) Between Measures

\begin{tabular}{|c|c|c|c|c|c|c|c|c|c|c|}
\hline & ODI & VAS & FAB-pa & FAB-w & PCS & HADS-a & HADS-d & FSE & SF-I 2 (PCS) & SF-I 2 (MCS) \\
\hline ODI & I & $0.328 * *$ & $0.240^{* *}$ & $0.474 * *$ & $0.4 \mid I^{* *}$ & $0.468^{* *}$ & $0.44 I^{* *}$ & $-0.213 * *$ & $-0.217 * *$ & $-0.164 *$ \\
\hline VAS & & 1 & $0.627^{* *}$ & $0.690 * *$ & $0.684^{* *}$ & $0.263^{* *}$ & $0.23 I^{* *}$ & $0.386 * *$ & -0.137 & $-0.189 *$ \\
\hline FAB-pa & & & 1 & $0.605^{* *}$ & 0.590 ** & 0.126 & 0.106 & $0.318^{* *}$ & $-0.238 * *$ & 0.032 \\
\hline FAB-w & & & & I & $0.727^{* *}$ & $0.366^{* *}$ & $0.297^{* *}$ & $0.242^{* *}$ & -0.090 & -0.114 \\
\hline PCS & & & & & 1 & $0.378^{* *}$ & $0.299 * *$ & $0.404 * *$ & -0.135 & -0.160 \\
\hline HADS-a & & & & & & 1 & $0.494 * *$ & -0.106 & $-0.236 * *$ & -0.124 \\
\hline HADS-d & & & & & & & I & -0.133 & $-0.285^{* *}$ & $-0.273 * *$ \\
\hline FSE & & & & & & & & I & $0.180 *$ & 0.157 \\
\hline SF-I 2 (PCS) & & & & & & & & & I & -0.001 \\
\hline SF-I2 (MCS) & & & & & & & & & & I \\
\hline
\end{tabular}

Notes: $*$ is $p<0.05$, and $* *$ is $p<0.01$.

Abbreviations: FABQ-pa, Fear Avoidance Beliefs Questionnaire-physical activity; FABQ-w, Fear Avoidance Beliefs Questionnaire-work; FSE, Functional Self-Efficacy Scale; HADS-a, Hospital Anxiety and Depression Scale-anxiety; HADS-d, Hospital Anxiety and Depression Scale-depression; ODI, Oswestry Disability Index; PCS, Pain Catastrophizing Scale; SF-12 (MCS), Short form-12 questionnaire (mental component scale); SF-12 (PCS), Short form- 12 (physical component scale); VAS, visual analog scale.

\section{Validation of Measures}

The outcomes of the study indicated that the Urdu versions of FABQ, PCS, HADS, FSE, ODI, and SF-12 are valid and reliable tools for assessment of psychological risk factors (fear-avoidance beliefs and catastrophic thoughts), psychological distress (anxiety and depression), functional self-efficacy, disability, and generic health-related quality of life in the Urdu-speaking population. These Urdu 
Table 5 Total Effect, Direct Effect, Indirect Effect, and R $^{2}$ Values for the Mediation Model of Pain (VAS) with Disability (ODI)

\begin{tabular}{|l|l|l|l|l|l|l|l|}
\hline Model & Path & $\mathbf{B}$ & $\mathbf{9 5 \%} \mathbf{C l}$ & $\mathbf{S E}$ & t Score & $\boldsymbol{p}$-value & Model $\boldsymbol{r}^{\mathbf{2}}$ \\
\hline VAS to ODI & Total effect (c) & 2.36 & 1.26 to 3.46 & 0.56 & 4.24 & $<0.001$ & 0.108 \\
& Direct effect (c') & 0.54 & -0.90 to 1.98 & 0.73 & 0.75 & 0.456 & \\
& Indirect effect $(\mathrm{a} \times \mathrm{b})$ & 1.82 & $0.5 \mathrm{I}$ to 3.32 & 0.72 & & & \\
\hline
\end{tabular}

Abbreviations: $B$, unstandardized beta; $\mathrm{Cl}$, confidence interval; ODI, Oswestry Disability Index; SE, standard error; VAS, visual analog scale.

Table 6 Indirect Effects of Fear (FABQ-Pa, FABQ-w), Catastrophizing (PCS), Anxiety (HADS-A), Depression (HADS-d), HRQoL Mental Component (MCS), and Self-Efficacy (FSE) on the Relationship Between Pain (VAS) and Disability (ODI)

\begin{tabular}{|c|c|c|c|c|c|c|c|c|c|c|c|}
\hline & \multicolumn{4}{|c|}{ a Path (Pain to Mediator) } & \multicolumn{4}{|c|}{ b Path (Mediator on Disability) } & \multicolumn{3}{|c|}{ Indirect Effect ( $\mathbf{x} \times$ b Path) } \\
\hline & B & SE & t Score & p-value & B & SE & t Score & p-value & B & SE & $95 \% \mathrm{Cl}$ \\
\hline \multicolumn{12}{|l|}{ VAS to ODI } \\
\hline FABQ-pa & 1.49 & 0.15 & 9.84 & $<0.001$ & -0.06 & 0.27 & -0.23 & 0.817 & -0.09 & 0.41 & -0.87 to 0.73 \\
\hline FABQ-w & 3.43 & 0.29 & 11.63 & $<0.001$ & 0.36 & 0.15 & 2.44 & 0.016 & 1.25 & 0.51 & 0.30 to 2.30 \\
\hline PCS & 3.87 & 0.34 & 11.44 & $<0.001$ & 0.29 & 0.14 & 2.12 & 0.035 & 1.13 & 0.55 & 0.12 to 2.28 \\
\hline HADS-a & 0.37 & 0.11 & 3.33 & $<0.001$ & 0.79 & 0.39 & 2.01 & 0.047 & 0.29 & 0.17 & -0.001 to 0.67 \\
\hline HADS-d & 0.33 & 0.11 & 2.90 & 0.004 & 0.86 & 0.38 & 2.24 & 0.026 & 0.28 & 0.15 & 0.03 to 0.61 \\
\hline FSE & 2.44 & 0.48 & 5.11 & $<0.001$ & -0.40 & 0.09 & -4.64 & $<0.001$ & -0.99 & 0.29 & -1.61 to -0.49 \\
\hline SF-I2 (MCS-I2) & -0.59 & 0.25 & -2.35 & 0.02 & 0.84 & 0.16 & 0.54 & 0.589 & -0.50 & 0.96 & -0.26 to 0.13 \\
\hline
\end{tabular}

Abbreviations: B, unstandardized beta; Cl, confidence interval; FABQ-pa, Fear Avoidance Beliefs Questionnaire-physical activity; FABQ-w, Fear Avoidance Beliefs Questionnaire-work; FSE, Functional Self-Efficacy Scale; HADS-a, Hospital Anxiety and Depression Scale-anxiety; HADS-d, Hospital Anxiety and Depression Scaledepression; SF-I 2 (MCS), Short form-12 questionnaire (mental component scale); SE, standard error; ODI, Oswestry Disability Index; PCS, Pain Catastrophizing Scale; VAS, visual analog scale.

versions were easy to understand by participants and low cost for use in both clinical settings and future research. There is an absence of "gold standard" measures to evaluate the questionnaires for criterion validity; thus, the correlations between variables were compared for convergent construct validity.

The Urdu versions of FABQ and PCS are consistent (FABQ-p $\alpha=0.88$, FABQ-w $\alpha=0.94$, PCS helplessness $\alpha=0.93$, PCS magnification $\alpha=0.88$, PCS rumination $\alpha=0.89$ ) and reliable (FABQ-p $r=0.90$, FABQ-w $r=0.85$, PCS $r=0.95$ ) tools for assessment of psychosocial risk factors associated with CLBP and disability. The internal consistency and reliability coefficients were found in this study for FABQ. Past studies testing cross-culturally adapted Brazilian-Portuguese, Finnish, Arabic, Persian, and Turkish FABQ versions reported good internal consistency (FABQ-p $\alpha=0.71-0.94$, FABQ-w $\alpha=0.74-0.89$ ) and reliability (FABQp $r=0.73-0.97$, FABQ-w $r=0.74-0.89) .^{24,27,30-32}$

The current study found good internal consistency $(\alpha=0.69$ and 0.69 ) for Urdu versions of HADS-a and HADS-d, respectively, and the correlation between both subscales was $0.494(p<0.001)$. The results of this study are consistent with the past studies of cross-cultural adaptation of HADS into Swedish, Italian, German, French, Spanish, Chinese, and Arabic languages. ${ }^{46}$ A literature review on validation studies of HADS found the values for internal consistency from Cronbach's $\alpha=0.68$ to 0.93 for HADS-anxiety and $\alpha=0.67$ to.90 for HADSdepression subscales. ${ }^{46}$ HADS had a between subscale mean correlation of 0.82 (0.57 to 0.90$)$.

Several past studies have found the internal consistency of ODI from $\alpha=0.71$ to 0.87 and test-retest reliability between $r=0.83$ and $0.99 .^{39}$ The psychometric outcomes of the Urdu version of ODI in this study (internal consistency $\alpha=0.84$, reliability $r=0.98$ ) are comparable to the ODI versions in Danish, Dutch, Finnish, German, Greek, French, Spanish, and Norwegian languages. ${ }^{39}$

\section{Fear-Avoidance Model in Pakistan}

The results of this study support previous research for the mediating role of catastrophizing, psychological distress (ie, depression and anxiety), and self-efficacy on the relationship between pain and disability in people with CLBP, ${ }^{11,56}$ and extends these findings to the Pakistani population. Moreover, these findings provide a unique contrast to previous research in that fear-avoidance beliefs 
regarding work, but not physical activity, were a significant mediator of the relationship between pain and disability. For example, in a recent cross-sectional study of 218 people having CLBP in Australia ${ }^{56}$ the indirect effect of fear-avoidance beliefs about activity $(B=0.51,95 \% \mathrm{CI}$ 0.25 to 0.85 ) was a mediator of the association between pain and disability, whereas fear-avoidance beliefs about work did not meet the criteria for mediation on the $b$ path (fear on disability). A primary difference between the samples in the two studies, which may reflect overall socioeconomic differences between Australia and Pakistan, was the percentage of participants involved in paid employment. In the sample from Australia, ${ }^{56} 68 \%$ of individuals reported being in paid employment (part- or full-time) compared to $38 \%$ in the current study. Thus, while not statistically tested in either study, current work status may be a moderating variable that explains the effect of fear-avoidance beliefs on the relationship of pain with disability. Whether the lower level of paid employment reported in the current study is attributed to the original cause of pain occurring at work, thus explaining the strength of effect for fear-avoidance about work, was not examined here. Conversely, this result could be explained by the fear unemployed individuals with back pain have about work further worsening their symptoms, thus jeopardizing future employment status. Combined with this is the relative importance of work compared to general physical activity for a sample with higher unemployment, explaining why fear-avoidance about physical activity was not a significant mediator despite average scores $(17.2 \pm 5.8)$ comparable to values from AngloSaxon countries (eg, Australia, $13.8 \pm 5.6) .{ }^{56}$ Furthermore, the sample characteristics of the current study and past research may also explain why the fear of physical activity was not a significant mediator in Pakistan. For instance, the mean age of CLBP patients included in this study $(36 \pm 11.6)$ and duration of pain symptoms were comparable to previous studies (eg, mean age ranging from $31.4 \pm 12.1$ to $50.6 \pm 7.2) .{ }^{11}$ However, the average disability scores in the current sample are much higher (ODI: $34.6 \pm 17.4$ ). Likewise, the sample from Pakistan examined in this study had much higher mean scores for work-related fear and catastrophizing (FABQ-w: $26.3 \pm 12.1$, PCS: $33.9 \pm 13.7$ ) than Australian $^{56}$ (FABQ-w: $11.3 \pm 9.8$, PCS: $15.6 \pm 12.6$ ) and United States ${ }^{57}$ (FABQ-w: $9.8 \pm 7.5$, PCS: $14.7 \pm$ 10.9) samples. While we cannot explain the differential relationship of fear-avoidance beliefs about activity and work observed in this study, this data suggest that employment status should be considered as a moderating variable in future studies of the fear-avoidance model.

\section{Limitations}

As a cross-sectional study, temporal causality between the different measures in this study cannot be established. Future prospective studies are needed to further test the causal relationships within fear-avoidance model in Pakistan. Dependence of data collection on an internet-based survey and self-report measures may have risks of response-related biases. The penetration rate of the internet in Pakistan is $31.19 \%$ as reported by the Pakistan Telecommunications Authority. ${ }^{58}$ However, the number of internet subscribers in Pakistan (65.13 million) is far more than the total population of Australia and New Zealand combined. Also, the questionnaires with good clinimetric characteristics were utilized to reduce the risk of response biases. Thus, the current results likely have good generalizability for clinical practice. While this study was not sufficiently powered to detect significant indirect effects with small $\mathrm{a}$ or $\mathrm{b}$ paths (ie, $\mathrm{B}=0.20$ ), two mediators did not reach significance in the $b$ path (FABQ-pa and MCS-12) and the only mediator that had a negligible effect in the $\mathrm{b}$ path $(B=-0.03)$ was FABQ-pa. Finally, as a study aimed to translate and provide preliminary evidence for the fear-avoidance model in people with CLBP from Pakistan, we did not design this study to explore potential moderators of the pathways examined here (eg, structured physical activity, work status).

\section{Conclusions}

The outcomes of the study indicate that the Urdu versions of FABQ, PCS, HADS, FSE, ODI, and SF-12 are valid and dependable tools to measure fear-avoidance beliefs, catastrophic thoughts, anxiety, depression, self-efficacy, pain-related disability, and generic health-related quality of life in the Urdu-speaking population. The current findings support previous literature for the mediation effect of fear-avoidance beliefs, catastrophizing, psychological distress, and self-efficacy on the association between pain and disability in people with CLBP, and indicate the work status should be considered as a potential moderating variable in future analyses.

\section{Data Sharing Statement}

The datasets used and/or analyzed during the current study and Urdu version of measures are available from the corresponding author on reasonable request. 


\section{Disclosure}

The authors declare that they have no competing interests. The authors received no specific funding for this work.

\section{References}

1. Murray CJL, Lopez AD. Measuring the global burden of disease. New Eng J Med. 2013;369(5):448-457. doi:10.1056/NEJMra1201534

2. Dagenais S, Caro J, Haldeman S. A systematic review of low back pain cost of illness studies in the United States and internationally. Spine J. 2008;8(1):8-20. doi:10.1016/j.spinee.2007.10.005

3. Bishwajit G, Tang S, Yaya S, Feng Z. Participation in physical activity and back pain among an elderly population in South Asia. J Pain Res. 2017;10:905-913. doi:10.2147/JPR.S133013

4. Stankov L. Conservative syndrome: individual and cross-cultural differences. J Cross Cult Psych. 2017;48(6):950-960. doi:10.1177/ 0022022117709984

5. Hurwitz E, Randhawa K, Torres $P$, et al. The global spine care initiative: a systematic review of individual and community-based burden of spinal disorders in rural populations in low- and middleincome communities. Eur Spine J. 2018;27(S6):802-815. doi:10.1007/s00586-017-5393-z

6. Louw QA, Morris LD, Grimmer-Somers K. The prevalence of low back pain in Africa: a systematic review. BMC Musculoskel Disord. 2007;8:105. doi:10.1186/1471-2474-8-105

7. Volinn E. The epidemiology of low back pain in the rest of the world: a review of surveys in low- and middle-income countries. Spine. 1997;22(15):1747-1754. doi:10.1097/00007632-199708010-00013

8. Nicholas MK, Linton SJ, Watson PJ, Main CJ; Group tDotFW. Early identification and management of psychological risk factors ("yellow flags") in patients with low back pain: a reappraisal. Phys Ther. 2011;91(5):737-753.

9. Turner JA, Jensen MP, Romano JM. Do beliefs, coping, and catastrophizing independently predict functioning in patients with chronic pain? Pain. 2000;85(1-2):115-125. doi:10.1016/S0304-3959(99)00259-6

10. Vlaeyen J, Linton S. Fear-avoidance and its consequences in chronic musculoskeletal pain: a state of the art. Pain. 2000;85(3):317-332. doi:10.1016/S0304-3959(99)00242-0

11. Lee H, Hubscher M, Moseley LG, et al. How does pain lead to disability? A systematic review and meta-analysis of mediation studies in people with back and neck pain. Pain. 2015;156(6):988-997. doi:10.1097/j.pain.0000000000000146

12. Smeets RJEM, Vlaeyen JWS, Kester ADM, Knottnerus JA. Reduction of pain catastrophizing mediates the outcome of both physical and cognitive-behavioral treatment in chronic low back pain. J Pain. 2006;7(4):261-271. doi:10.1016/j.jpain.2005.10.011

13. Marshall PWM, Kennedy S, Brooks C, Lonsdale C. Pilates exercise or stationary cycling for chronic nonspecific low back pain: does it matter? Spine. 2013;38(15):E952-9. doi:10.1097/BRS.0b013e318 $297 \mathrm{c} 1 \mathrm{e} 5$

14. Lethem J, Slade PD, Troup JD, Bentley G. Outline of a fear-avoidance model of exaggerated pain perception. Behav Res Ther. 1983;21:401-408. doi:10.1016/0005-7967(83)90009-8

15. Kovacs F, Abraira V, Cano A, et al. Fear avoidance beliefs do not influence disability and quality of life in Spanish elderly subjects with low back pain. Spine. 2007;32(19):2133-2138.

16. Kovacs F, Seco J, Royuela A, Pena A, Muriel A. The correlation between pain, catastrophizing, and disability in subacute and chronic low back pain. Spine. 2011;36:339-345. doi:10.1097/BRS.0b013e3181cfba29

17. Kovacs FM, Seco J, Royuela A, Corcoll-Reixach J, Pena-Arrebola A, Network SBPR. The prognostic value of catastrophizing for predicting the clinical evolution of low back pain patients: a study in routine clinical practice within the Spanish National Health Service. Spine J. 2012;12(7):545-555. doi:10.1016/j.spinee.2012.06.002
18. Burton AK, Waddell G, Tillotson KM, Summerton N. Information and advice to patients with back pain can have a positive effect. A randomized controlled trial of a novel educational booklet in primary care. Spine. 1999;24(23):2484-2491.

19. O’Keefe M, O’Sullivan P, Purtill H, Bargary N, O’Sullivan K. Cognitive functional therapy compared with a group-based exercise and education intervention for chronic low back pain: a multicentre randomised controlled trial (RCT). Br J Sports Med. 2019.

20. Vibe Fersum K, O'Sullivan P, Skouen JS, Smith A, Kvale A. Efficacy of classification-based cognitive functional therapy in patients with non-specific chronic low back pain: a randomized controlled trial. Eur J Pain. 2013;17:916-928. doi:10.1002/j.15322149.2012.00252.x

21. Kovacs FM, Llobera J, Abriaira V, et al. Effectiveness and costeffectiveness analysis of neuroreflexotherapy for subacute and chronic low back pain in routine general practice: a cluster randomized, controlled trial. Spine. 2002;27(11):1149-1159. doi:10.1097/ 00007632-200206010-00004

22. Urrutia G, Burton AK, Morral A, Bonfill X, Zanoli G. Neuroreflexotherapy for non-specific low-back pain. Cochrane Database Syst Rev. 2004;2(CD003009).

23. Chapman JR, Norvell DC, Hermsmeyer JT, et al. Evaluating common outcomes for measuring treatment success for chronic low back pain. Spine. 2011;36:S54-S68. doi:10.1097/BRS.0b013e31822ef74d

24. De Souza SF, Da Silva Marinho BC, Siqueira GF, Maher OPC, Costa OPL. Psychometric testing confirms that the Brazilian-Portuguese adaptations, the original versions of the fear-avoidance beliefs questionnaire, and the Tampa scale of kinesiophobia have similar measurement properties. Spine. 2008;33(9):1028-1033. doi:10.1097/ BRS.0b013e31816c8329

25. Kaka B, Ogwumike OO, Idowu OA, et al. Translation of the fear avoidance beliefs questionnaire into Hausa language. Glob $\mathrm{J}$ Health Sci. 2015;7(3):116-123.

26. Igwesi-Chidobe $\mathrm{CN}$, Obiekwe C, Sorinola IO, Godfrey EL. Assessing self-reported disability in a low-literate population with chronic low back pain: cross-cultural adaptation and psychometric testing of Igbo Roland Morris disability questionnaire. Disabil Rehabil. 2019;41(8):948-957. doi:10.1080/09638288.2017.1416185

27. Terho H, Haapea M, Paananen M, Korniloff K, Häkkinen A, Karppinen J. Translation and validation of the Finnish version of the fear-avoidance beliefs questionnaire (FABQ). Scandinavian $J$ Pain. 2016;10(1):113-118. doi:10.1016/j.sjpain.2015.09.003

28. Chaory K, Fayad F, Rannou F, et al. Validation of the French version of the fear avoidance belief questionnaire. Spine. 2004;29 (8):908-913.

29. Denteneer L, Van Daele U, Truijen S, et al. The modified low back pain disability questionnaire: reliability, validity, and responsiveness of a Dutch language version. Spine. 2018;43(5):E292-E8. doi:10.1097/BRS.0000000000002304

30. Alanzai F, Gleeson P, Olson S, Roddey T. Translation and validation fo the Arabic version of the fear-avoidance beliefs questionnaire in patients with low back pain. Spine. 2017;42(7):E411-E6. doi:10.1097/BRS.0000000000001802

31. Korkmaz N, Akinci A, Yorukan S, Surucu H, Saracbasi O, Ozcakar L. Validation and reliability of the Turkish version of the fear avoidance beliefs questionnaire in patients with low back pain. Eur J Phys Med Rehabil Med. 2009;527-535.

32. Rostami M, Noorian N, Mansournia MA, Sharafi E, Babaki AES, Kordi R. Validation of the Persian version of the fear avoidance belief questionnaire in patients with low back pain. J Back Musculoskelet Rehabil. 2014;27(2):213-221. doi:10.3233/BMR-130439

33. Worldbank. Available from: http://documents.worldbank.org/curated/ en/619971467987825539/pdf/100119-WP-PUBLIC-Box393225BPakistan-country-snapshot.pdf. 2019 Accessed February 26, 2020.

34. Muhammad Ashar M, Muhammad W. Economics of health and health care in Pakistan. J Pakistan Med Assoc. 2013;63(7):814-815. 
35. Terwee C, Mokkink L, Knol D, Ostelo R, Bouter L, Vet H. Rating the methodological quality in systematic reviews of studies on measurement properties: a scoring system for the COSMIN checklist. Qual Life Res. 2012;21(4):651-657. doi:10.1007/s11136-011-9960-1

36. Fritz MS, Mackinnon DP. Required sample size to detect the mediated effect. Psychol Sci. 2007;18(3):233-239. doi:10.1111/ j.1467-9280.2007.01882.x

37. Beaton ED, Bombardier EC, Guillemin EF, Ferraz EMB. Guidelines for the process of cross-cultural adaptation of self-report measures. Spine. 2000;25(24):3186-3191. doi:10.1097/00007632-20001215000014

38. Jensen MP, Turner AJ, Romano MJ, Fisher DL. Comparative reliability and validity of chronic pain intensity measures. Pain. 1999;83 (2):157-162. doi:10.1016/S0304-3959(99)00101-3

39. Fairbank JC, Pynsent PB. The Oswestry disability index. Spine. 2000;25(22):2940-2953. doi:10.1097/00007632-200011150-00017

40. Waddell G, Newton M, Henderson I, Somerville D, Main CJ. A fearavoidance beliefs questionnaire (FABQ) and the role of fear-avoidance beliefs in chronic low back pain and disability. Pain. 1993;52 (2):157-168. doi:10.1016/0304-3959(93)90127-B

41. Swinkels-Meewisse EJCM, Swinkels RAHM, Verbeek ALM, Vlaeyen JWS, Oostendorp RAB. Psychometric properties of the Tampa scale for kinesiophobia and the fear-avoidance beliefs questionnaire in acute low back pain. Man Ther. 2003;8(1):29-36 doi:10.1054/math.2002.0484

42. Sullivan MJL, Bishop SR. The pain catastrophizing scale: development and validation. Psychol Assess. 1995;7(4):524-532. doi:10.1037/1040-3590.7.4.524

43. Lame IE, Peters ML, Kessels AG, Van Kleef M, Patijn J. Test-retest stability of the pain catastrophizing scale and the Tampa scale for kinesiophobia in chronic pain patients over a longer period of time. $J$ Health Psych. 2008;13:820-826. doi:10.1177/1359105308093866

44. Van Damme S, Crombez G, Bijttebier P, Goubert L, van Houdenhove B. A confirmatory factor analysis of the pain catastrophizing scale: invariant factor structure across clinical and non-clinical populations. Pain. 2002;96:319-324. doi:10.1016/S0304-3959(01)00463-8

45. Zigmond AS, Snaith RP. The hospital anxiety and depression scale. Acta Psychiatr Scan. 1983;67:361-370. doi:10.1111/j.16000447.1983.tb09716.x

46. Bjelland I, Dahl AA, Haug TT, Neckelmann D. The validity of the hospital anxiety and depression scale: an updated literature review. $J$ Psychosomatic Res. 2002;52:69-77. doi:10.1016/S0022-3999(01) 00296-3
47. Anderson KO, Dowds BN, Pelletz RE, Edwards WT, PeetersAsdourian C. Development and initial validation of a scale to measure self-efficacy beliefs in patients with chronic pain. Pain $1995 ; 63$ (1):77-84. doi:10.1016/0304-3959(95)00021-J

48. Woby SR, Roach NK, Urmston M, Watson PJ. The relation between cognitive factors and levels of pain and disability in chronic low back pain patients presenting for physiotherapy. Eur J Pain. 2007;11 (8):869-877. doi:10.1016/j.ejpain.2007.01.005

49. Woby SR, Urmston M, Watson PJ. Self-efficacy mediates the relation between pain-related fear and outcome in chronic low back pain patients. Eur J Pain. 2007;11(7):711-718. doi:10.1016/j. ejpain.2006.10.009

50. Ware JE, Kosinski M, Keller SD. A 12-Item Short-Form Health Survey: construction of scales and preliminary tests of reliability and validity. Med Care. 1996;34(3):220-233. doi:10.1097/ 00005650-199603000-00003

51. Luo X, Lynn George M, Kakouras I, et al. Reliability, validity, and responsiveness of the short form 12-item survey (SF-12) in patients with pack Pain. Spine. 2003;28(15):1739-1745. doi:10.1097/01. BRS.0000083169.58671.96

52. Terwee CB, Bot SD, de Boer MR, et al. Quality criteria were proposed for measurement properties of health status questionnaires. $J$ Clin Epidemiol. 2007;60(1):34-42.

53. Cheung GW, Lau RS. Testing mediation and suppression effects of latent variables: bootstrapping with structural equation models. Organizational Res Meth. 2008;11:296-325. doi:10.1177/ 1094428107300343

54. Preacher KJ, Hayes AF. Asymptotic and resampling strategies for assessing and comparing indirect effects in multiple mediator models. Behav Research Meth. 2008;40:879-891. doi:10.3758/BRM.40.3.879

55. Tabachnick B, Fiddell L. Using Multivariate Statistics. Boston, MA Pearson; 2013.

56. Marshall PWM, Schabrun S, Knox MF. Physical activity and the mediating effect of fear, depression, anxiety, and catastrophizing on pain related disability in people with chronic low back pain. PLoS One. 2017;12(7):e0180788. doi:10.1371/journal.pone.0180788

57. Gay CW, Horn ME, Bishop MD, Robinson ME, Bialosky JE. Investigating dynamic pain sensitivity in the context of the fearavoidance model. Eur J Pain. 2015;19(1):48-58. doi:10.1002/ejp.519

58. Pakistan Telecommunication Authority. Available from:https://www. pta.gov.pk/en/media-center/single-media/ptas-response-to-hootsuitesdigital-2019-pakistan-report-210619. Accessed 26 February 2020.
Journal of Pain Research

\section{Publish your work in this journal}

The Journal of Pain Research is an international, peer reviewed, open access, online journal that welcomes laboratory and clinical findings in the fields of pain research and the prevention and management of pain. Original research, reviews, symposium reports, hypothesis formation and commentaries are all considered for publication. The manuscript

Submit your manuscript here: https://www.dovepress.com/journal-of-pain-research-journal management system is completely online and includes a very quick and fair peer-review system, which is all easy to use. Visit http:// www.dovepress.com/testimonials.php to read real quotes from published authors. 